\title{
SEQUENCES OF DIFFERENTIAL SYSTEMS
}

\author{
MAKOTO NAGATA \\ (Communicated by Dennis A. Hejhal)
}

\begin{abstract}
Our main result deals with certain properties of the sizes of a differential system and its dual system. It represents an improvement of part of the results in André's G-function theory (Max-Planck-Institut, Bonn, 1989).
\end{abstract}

Let $K$ be a number field which is a finite extension of $\mathbb{Q}$.

We consider two differential systems:

$$
(\mathcal{D}-A) \bar{y}=0
$$

and the dual system of (0.1),

$$
\left(\mathcal{D}+{ }^{t} A\right) \bar{z}=0
$$

where $A \in M_{n}(K(x))$ and where $\mathcal{D}$ denotes the differential operator $\mathcal{D}:=\frac{d}{d x}$.

Our aim in this paper will be to consider properties of two sequences which are defined using (0.1) and (0.2), and to then show that the size of (0.1) (cf. definition below) and the size of (0.2) are equal to each other.

We normalize every place $v$ of $K$ as in [1]. We extend a non-Archimedean valuation $|\cdot|_{v}$ on $K$ to a valuation on $K(x)$ as the Gauss absolute value (cf. [1, Ch. IV]). We also define a pseudovaluation on the set of $n \times l$-matrices of rational functions, $M_{n, l}(K(x))$, in the usual way; i.e., for $M=\left(f_{i, j}(x)\right)_{j=1, \ldots, l}^{i=1, \ldots, n} \in M_{n, l}(K(x))$,

$$
|M|_{v}:=\max _{\substack{i=1, \ldots, n \\ j=1, \ldots, l}}\left|f_{i, j}(x)\right|_{v}
$$

For a sequence $\left\{F_{i}\right\} \subset M_{n, l}(K(x))$, we define the size of $\left\{F_{i}\right\}$ as

$$
\sigma\left(\left\{F_{i}\right\}\right):=\varlimsup_{m \rightarrow \infty} \sum_{v \nmid \infty} \frac{1}{m} \max _{i \leq m} \log \left(\max \left(1,\left|F_{i}\right|_{v}\right)\right)
$$

and the global radius of $\left\{F_{i}\right\}$ by

$$
\rho\left(\left\{F_{i}\right\}\right):=\sum_{v \nmid \infty} \varlimsup_{m \rightarrow \infty} \frac{1}{m} \max _{i \leq m} \log \left(\max \left(1,\left|F_{i}\right|_{v}\right)\right),
$$

Received by the editors October 25, 1993 and, in revised form, July 15, 1994

1991 Mathematics Subject Classification. Primary 12H25; Secondary 11S99.

Key words and phrases. G-functions.

(C)1996 American Mathematical Society 
where $\sum_{v \nmid \infty}$ means that $v$ ranges over non-Archimedean valuations.

We further define two sequences $\left\{A_{i}\right\}$ and $\left\{A_{i}^{*}\right\} \subset M_{n}(K(x))$ as follows:

$$
A_{0}=A_{0}^{*}=I \quad(I \text { is the identity matrix })
$$

and for $i=0,1, \ldots$, recursively

$$
\left\{\begin{array}{l}
A_{i+1}=\frac{1}{i+1}\left(\mathcal{D} A_{i}+A_{i} A\right), \\
A_{i+1}^{*}=\frac{1}{i+1}\left(\mathcal{D} A_{i}^{*}-A_{i}^{* t} A\right),
\end{array}\right.
$$

where $A$ is as in the differential system $(0.1)$.

Let us write simply $\sigma(A)$ (resp. $\sigma\left(A^{*}\right), \rho(A), \rho\left(A^{*}\right)$ ) instead of $\sigma\left(\left\{A_{i}\right\}\right)$ (resp. $\left.\sigma\left(\left\{A_{i}^{*}\right\}\right), \rho\left(\left\{A_{i}\right\}\right), \rho\left(\left\{A_{i}^{*}\right\}\right)\right)$.

Following André and Bombieri, we call $\sigma(A)$ (resp. $\left.\sigma\left(A^{*}\right)\right)$ the size of the differential system (0.1) (resp. $(0.2))$ and $\rho(A)$ (resp. $\rho\left(A^{*}\right)$ ) the global radius of $(0.1)$ (resp. (0.2)); cf. [1], [2].

Remark 1. One can see that $\sigma(A)$ (resp. $\rho(A)$ ) is equal to the size (resp. the global radius) of the $\partial$-module over $K(x)$ in [1].

We now consider the relations among two sequences which are defined as follows.

Suppose that $P$ and $R$ are elements in the set of $n \times l$-matrices of differentiable functions, $M_{n, l}\left(C^{\infty}\right)$. We define two sequences $\left\{P^{\langle i\rangle}\right\}$ and $\left\{R^{\langle i\rangle}\right\} \subset M_{n, l}\left(C^{\infty}\right)$ via

$$
\left\{\begin{array}{l}
P^{\langle 0\rangle}=P \\
R^{\langle 0\rangle}=R
\end{array}\right.
$$

and for $i=0,1, \ldots$, recursively

$$
\left\{\begin{array}{l}
P^{\langle i+1\rangle}=\frac{1}{i+1}\left(\mathcal{D} P^{\langle i\rangle}+{ }^{t} A P^{\langle i\rangle}\right), \\
R^{\langle i+1\rangle}=\frac{1}{i+1}\left(\mathcal{D} R^{\langle i\rangle}-A R^{\langle i\rangle}\right) .
\end{array}\right.
$$

Remark 2. These sequences, $\left\{P^{\langle i\rangle}\right\}$ and $\left\{R^{\langle i\rangle}\right\}$, appear in many papers which are the applications of Padé-type approximations to G-function and E-function theories (cf. [1], [2], [3], [4], [5], [7], [8], etc.).

Lemma 3. For $i, j, k=0,1, \ldots$, one has

$$
\begin{aligned}
& \frac{\mathcal{D}^{k}}{k !}\left({ }^{t} P^{\langle 0\rangle} R^{\langle j\rangle}\right)=\sum_{s=0}^{k}\left(\begin{array}{c}
j+k-s \\
j
\end{array}\right){ }^{t} P^{\langle s\rangle} R^{\langle j+k-s\rangle}, \\
& \frac{\mathcal{D}^{k}}{k !}\left({ }^{t} P^{\langle i\rangle} R^{\langle 0\rangle}\right)=\sum_{s=0}^{k}\left(\begin{array}{c}
i+k-s \\
i
\end{array}\right){ }^{t} P^{\langle i+k-s\rangle} R^{\langle s\rangle} .
\end{aligned}
$$

Proof. We prove the identity (3.1) for any $j$ by induction on $k$. For any $j=0,1, \ldots$, the identity (3.1) is clear for $k=0$. Assume then that (3.1) is true for any $j$ for a 
given $k \geq 0$. We prove it for $k+1$. For any $j=0,1, \ldots$,

$$
\begin{aligned}
D^{k+1}\left({ }^{t} P^{\langle 0\rangle} R^{\langle j\rangle}\right)= & \mathcal{D}\left(\mathcal{D}^{k}\left({ }^{t} P^{\langle 0\rangle} R^{\langle j\rangle}\right)\right)=k ! \sum_{s=0}^{k}\left(\begin{array}{c}
j+k-s \\
j
\end{array}\right) \mathcal{D}\left({ }^{t} P^{\langle s\rangle} R^{\langle j+k-s\rangle}\right) \\
= & \sum_{s=0}^{k} k !\left(\begin{array}{c}
j+k-s \\
j
\end{array}\right)(s+1){ }^{t} P^{\langle s+1\rangle} R^{\langle j+k-s\rangle} \\
& +\sum_{s=0}^{k} k !\left(\begin{array}{c}
j+k-s \\
j
\end{array}\right)(j+k-s+1){ }^{t} P^{\langle s\rangle} R^{\langle j+k-s+1\rangle} \\
= & \sum_{s=1}^{k+1} \frac{k !(j+k-s+1) !}{(k-s+1) ! j !} s^{t} P^{\langle s\rangle} R^{\langle j+k-s+1\rangle} \\
& +\sum_{s=0}^{k} \frac{k !(j+k-s+1) !}{(k-s) ! j !}{ }^{t} P^{\langle s\rangle} R^{\langle j+k+1-s\rangle} \\
= & (k+1) ! \sum_{s=0}^{k+1}\left(\begin{array}{c}
j+k+1-s \\
j
\end{array}\right){ }^{t} P^{\langle s\rangle} R^{\langle j+k+1-s\rangle} .
\end{aligned}
$$

Identity (3.2) is obtained by a similar argument.

Theorem 4. For $m=0,1, \ldots$, we have

$$
\begin{aligned}
& { }^{t} P^{\langle m\rangle} R^{\langle 0\rangle}=\sum_{i=0}^{m}(-1)^{m-i} \frac{\mathcal{D}^{i}}{i !}\left({ }^{t} P^{\langle 0\rangle} R^{\langle m-i\rangle}\right), \\
& { }^{t} P^{\langle 0\rangle} R^{\langle m\rangle}=\sum_{i=0}^{m}(-1)^{m-i} \frac{\mathcal{D}^{i}}{i !}\left({ }^{t} P^{\langle m-i\rangle} R^{\langle 0\rangle}\right) .
\end{aligned}
$$

Proof. We prove only (4.1). The argument for (4.2) is similar.

At first, one finds the equation

$$
\begin{aligned}
& \sum_{i=0}^{m-1}{ }^{t} P^{\langle i\rangle} R^{\langle m-i\rangle} \sum_{j=i}^{m-1}(-1)^{m-j}\left(\begin{array}{c}
m-i \\
j-i
\end{array}\right) \\
& =\sum_{i=0}^{m-1}(-1)^{m-i} \sum_{s=0}^{i}\left(\begin{array}{c}
m-s \\
i-s
\end{array}\right){ }^{t} P^{\langle s\rangle} R^{\langle m-s\rangle}
\end{aligned}
$$


by elementary calculations. We then have

$$
\begin{aligned}
& \frac{\mathcal{D}^{m}}{m !}\left({ }^{t} P^{\langle 0\rangle} R^{\langle 0\rangle}\right)={ }^{t} P^{\langle m\rangle} R^{\langle 0\rangle}+\sum_{i=0}^{m-1}{ }^{t} P^{\langle i\rangle} R^{\langle m-i\rangle} \quad \text { ( by Lemma 3 ) } \\
& \quad={ }^{t} P^{\langle m\rangle} R^{\langle 0\rangle}-\sum_{i=0}^{m-1}{ }^{t} P^{\langle i\rangle} R^{\langle m-i\rangle}\left(\sum_{j=0}^{m-i-1}\left(\begin{array}{c}
m-i \\
j
\end{array}\right)(-1)^{m-i-j}\right) \\
& \quad={ }^{t} P^{\langle m\rangle} R^{\langle 0\rangle}-\sum_{i=0}^{m-1}{ }^{t} P^{\langle i\rangle} R^{\langle m-i\rangle} \sum_{j=i}^{m-1}(-1)^{m-j}\left(\begin{array}{c}
m-i \\
j-i
\end{array}\right) \\
& \quad={ }^{t} P^{\langle m\rangle} R^{\langle 0\rangle}-\sum_{i=0}^{m-1}(-1)^{m-i} \sum_{s=0}^{i}\left(\begin{array}{c}
m-s \\
i-s
\end{array}\right){ }^{t} P^{\langle s\rangle} R^{\langle m-s\rangle} \quad \text { (by (4.3)) } \\
& \quad={ }^{t} P^{\langle m\rangle} R^{\langle 0\rangle}-\sum_{i=0}^{m-1}(-1)^{m-i} \frac{\mathcal{D}^{i}}{i !}\left({ }^{t} P^{\langle 0\rangle} R^{\langle m-i\rangle}\right)
\end{aligned}
$$

by the replacement of $k$ and $j$ in the identity (3.1) with $i$ and $m-i$. But this is equivalent to (4.1).

Corollary 5. For $m=0,1, \ldots$, one has

$$
\begin{aligned}
& A_{m}=\sum_{i=0}^{m}(-1)^{m-i} \frac{\mathcal{D}^{i}}{i !}{ }^{t} A_{m-i}^{*}, \\
& { }^{t} A_{m}^{*}=\sum_{i=0}^{m}(-1)^{m-i} \frac{\mathcal{D}^{i}}{i !} A_{m-i} .
\end{aligned}
$$

Proof. Simply replace both of $P$ and $R$ in Theorem 4 by $I$.

The following statement is the improvement of André's result [1, Ch. IV, §5.5].

Corollary 6. For the differential systems (0.1) and (0.2), we have

$$
\begin{gathered}
\sigma(A)=\sigma\left(A^{*}\right), \\
\rho(A)=\rho\left(A^{*}\right) .
\end{gathered}
$$

Proof. Suppose that $v$ is non-Archimedean. From the definition of the pseudovaluation on $M_{n}(K(x))$, it is clear that $\left|{ }^{t} A_{m}^{*}\right|_{v}=\left|A_{m}^{*}\right|_{v}$. By the identity (5.1), we have

$$
\left|A_{m}\right|_{v} \leq \max _{0 \leq i \leq m}\left|\frac{\mathcal{D}^{i}}{i !} A_{m-i}^{*}\right|_{v} \leq \max _{0 \leq i \leq m}\left|A_{i}^{*}\right|_{v}
$$

that is,

$$
\sigma(A) \leq \sigma\left(A^{*}\right)
$$

Similarly, by (5.2), we find

$$
\sigma(A) \geq \sigma\left(A^{*}\right)
$$

Therefore

$$
\sigma(A)=\sigma\left(A^{*}\right) .
$$

Identity (6.2) is proved by a similar argument.

Remark 7. Equation (6.2) is derived using a different method in [1, Ch. IV, $\S 3.3$, Lemma 2]. 


\section{REFERENCES}

1. Y. André, G-functions and Geometry, Max-Planck-Institut, Bonn, 1989. MR 90k:11087

2. E. Bombieri, On G-functions, Recent Progress in Analytic Number Theory, Vol. 2, Academic Press, New York, 1981, pp. 1-67. MR 83c:10050

3. D.V. Chudnovsky and G.V. Chudnovsky, Applications of Padé approximations to diophantine inequalities in values of G-functions, Lecture Notes in Math., vol. 1135, Springer-Verlag, Berlin, Heidelberg, and New York, 1985, pp. 9-51. MR 87a:11062

4. A.I. Galochkin, Estimates from below of polynomials in the values of analytic functions of a certain class, Math. USSR-Sb. 24 (1974), 385-407; Original article in Mat. Sb. 95 (137) (1974), 396-417.

5. X. Guangshan, On the arithmetic properties of G-functions, International Symposium in Memory of Hua Loo Keng (Number Theory), Springer-Verlag, Berlin, Heidelberg, and New York, 1991, pp. 331-346.

6. K. Mahler, Perfect systems, Compositio Math. 19 (1968), 95-166. MR 39:458

7. A.B. Shidlovskii, Transcendental numbers, Walter de Gruyter, Berlin and New York, 1989. MR 90j:11066

8. K. Väänänen, On linear forms of a certain class of $G$-functions and p-adic $G$-functions, Acta Arith. 36 (1980), 273-295. MR 82b:10045

Department of Mathematics, Tokyo institute of Technology, Oh-okayama, MEguro-Ku, TOKYO, 152, JAPAN

E-mail address: nagata@math.titech.ac.jp 